\title{
Vector Boson Mass Spectrum from Extra Dimension
}

\author{
Dao Vong Duc ${ }^{1}$, Nguyen Mong Giao ${ }^{2}$ \\ ${ }^{1}$ Institute of Physics, Hanoi, Vietnam \\ ${ }^{2}$ Institute of Physics, Ho Chi Minh City, Vietnam \\ Email: dvduc@iop.vast.ac.vn,nmgiao2011@yahoo.com.vn
}

Received April 29, 2013; revised May 31, 2013; accepted June 28, 2013

Copyright (C) 2013 Dao Vong Duc, Nguyen Mong Giao. This is an open access article distributed under the Creative Commons Attribution License, which permits unrestricted use, distribution, and reproduction in any medium, provided the original work is properly cited.

\begin{abstract}
Based on the mechanism for mass creation in the space-time with extradimensions proposed in our previous work, (arXiv: 1301.1405 [hep-th] 2013) we consider now the mass spectrum of vector bosons in extradimensions. It is shown that this spectrum is completely determined by some function of compactification length and closely related to the metric of extradimensions.
\end{abstract}

Keywords: Boson Mass Spectrum; Extra Dimensions

\section{Introduction}

The topology of space-time extradimensions has been a subject of intensive study in various aspects during the last time [1-8]. On the other side, the problem of particle masses, especially for gauge vector bosons, remains to be of actual character.

In our previous work [9] we have proposed a mechanism for the creation of particle masses based on the periodicity condition dictated from the compactification of extradimensions. In this approach the original field functions depend on both ordinary space-time coordinates and those for extradimensions, the ordinary field functions for ordinary 4-dimensional space-time are considered as effective field functions obtained by integration of the original ones over extraspace-time.

The mass of vector bosons has been treated in [9] for the simplest case with one extradimension. Along this line, in this work we extend the results to the general case for arbitrary number of extradimensions.

\section{Effective 4-Dimensional Vector Fields}

As in [9], we denote the $4+\mathrm{d}$ dimensional coordinate vector by $x^{M}$ with $M=\mu, 5,6, \cdots, 4+d$. The Greek indices will be used for conventional 4-dimensional Lorentz indices, $\mu=0,1,2,3$. For convenience we will use the notations $y^{a} \equiv x^{4+a}, a=1,2, \cdots, d$, and express the field function of coordinates as

$$
F\left(x^{M}\right) \equiv F\left(x^{\mu}, y^{a}\right) \equiv F(x, y)
$$

with the periodicity condition supposed to be of the form:

$$
F\left(x ; y^{a}+L^{(a)}\right)=f_{F}^{(a)} \cdot F(x, y)
$$

where $f_{F}^{(a)}$ is some parameter function depending on the compactification length $\mathrm{L}^{(\mathrm{a})}$.

The Condition (1) corresponds to the equation:

$$
\frac{\partial}{\partial y^{a}} f(x, y)=g_{F}^{(a)} \cdot F(x, y)
$$

with the relations:

$$
\begin{aligned}
& f_{F}^{(a)}=\mathrm{e}^{L^{(a)} \cdot g_{F}^{(a)}}, \\
& g_{F}^{(a)}=\frac{1}{L^{(a)}}\left[\ln f_{F}^{(a)}+2 \pi n i\right], n \in Z
\end{aligned}
$$

In general we can put:

$$
\begin{aligned}
& f_{F}^{(a)}=\rho_{F}^{(a)} \cdot \mathrm{e}^{\mathrm{i} \theta_{F}^{(a)}} \\
& g_{F}^{(a)}=\frac{1}{L^{(a)}}\left[\ln \rho_{F}^{(a)}+\mathrm{i}\left(\theta_{F}^{(a)}+2 \pi n\right)\right]
\end{aligned}
$$

where $\rho_{F}^{(a)}$ and $\theta_{F}^{(a)}$ are some functions of $L^{(a)}$.

For neutral field, $F^{+}=F, f_{F}^{(a)}$ is real and therefore $\theta_{F}^{(a)}=0, n=0$.

We now consider the neutral vector field $V_{M}(x, y)$ satisfying the periodicity condition.

$$
\begin{aligned}
& V_{M}\left(x, y^{a}+L^{(a)}\right)=f_{V_{M}}^{(a)} \cdot V_{M}(x, y) \\
& \frac{\partial}{\partial y^{a}} V_{M}(x, y)=g_{V_{M}}^{(a)} \cdot V_{M}(x, y)
\end{aligned}
$$


The free vector field $V_{M}(x, y)$ is described by the Lagrangian

$$
\begin{aligned}
L(x, y) & =-\frac{1}{4} F_{M N} F^{M N} \\
& =-\frac{1}{4} F_{\mu \nu} F^{\mu \nu}-\frac{1}{2} F_{\mu a} F^{\mu a}-\frac{1}{4} F_{a b} F^{a b}
\end{aligned}
$$

where

$$
\begin{aligned}
& F_{M N}=\frac{\partial V_{N}}{\partial x^{M}}-\frac{\partial V_{M}}{\partial x^{N}} \\
& F_{\mu v}=\frac{\partial V_{v}}{\partial x^{\mu}}-\frac{\partial V_{\mu}}{\partial x^{v}} \\
& F_{\mu a}=\frac{\partial V_{A}}{\partial x^{\mu}}-\frac{\partial V_{\mu}}{\partial y^{a}} \\
& F_{a b}=\frac{\partial V_{B}}{\partial y^{a}}-\frac{\partial V_{A}}{\partial y^{b}}
\end{aligned}
$$

Now let us consider the case when the field component $V_{A} \equiv V_{4+a}$ under treatement does not depend on $y^{b}$, unless $b=a$. In this case $F_{a b}=0$ and the Lagrangian (7) with Equation (6) taken into account becomes:

$$
\begin{aligned}
L(x, y) & =-\frac{1}{4} F_{\mu v} F^{\mu v} \\
& =-\frac{1}{2} \sum_{a=1}^{d} \eta_{a a}\left(\partial_{\mu} V_{A}-g_{V_{A}}^{(a)} V_{\mu}\right)\left(\partial^{\mu} V_{A}-g_{V_{A}}^{(a)} V^{\mu}\right)
\end{aligned}
$$

where $\eta_{a b}$ is Minkowski metric for extra dimensions.

Now we define a set of new physical vector fields $Z_{\mu}^{(a)}$ by putting

$$
Z_{\mu}^{(a)} \equiv V_{\mu}-\frac{1}{g_{V_{A}}^{(a)}} \partial_{\mu} V_{A}
$$

Note that the field strength tensor

$$
G_{\mu \nu}^{(a)} \equiv \partial_{\mu} Z_{v}^{(a)}-\partial_{\nu} Z_{\mu}^{(a)}
$$

remains unchanged and independent of $a$ :

$$
G_{\mu v}^{(a)} \equiv \partial_{\mu} Z_{v}-\partial_{\nu} V_{\mu} \equiv F_{\mu v}
$$

Therefore Equation (9) can be transformed into the form:

$$
\begin{aligned}
L(x, y)= & -\frac{1}{4} \sum_{a=1}^{d} \alpha(a) G_{\mu \nu}^{(a)} G^{(a) \mu v} \\
& -\frac{1}{2} \sum_{a} \eta_{a a} g_{V_{A}}^{(a)^{2}} Z_{\mu}^{(a)} Z^{(a) \mu}
\end{aligned}
$$

With the constraint:

$$
\sum_{a=1}^{d} \alpha(a)=1
$$

The Formula (13) tells that a set of $d$ vector fields in ordinary 4-dimensional space-time can be unified in a single vector field in the whole $(4+\mathrm{d})$-dimensional space-time with the distribution characterized by the coefficients obeying the constraint (14). This constraint is dictated by Equations (9)-(13). This result could be useful in the construction of Unifying models for gauge interactions.

\section{Equation of Motion and Mass Spectrum}

We start from the Lorentz invariant Lagrangian (13) and the effective action defined as:

$$
S=\int \mathrm{d} y S(y)
$$

Here, as in Section 2, for convenience we use the notation $y$ instead of $x$ for extradimension coordinate.

$$
S(y) \equiv \int \mathrm{d}^{4} x L(x, y)
$$

where $\int \mathrm{d} y$ means the integration over the extradimensions:

$$
\int \mathrm{d} y \equiv \int_{0}^{L^{(1)}} \int_{0}^{L^{(2)}} \cdots \int_{0}^{L^{(d)}} \mathrm{d} y^{1} \cdot \mathrm{d} y^{2}, \cdots, \cdot \mathrm{d} y^{d}
$$

The principle of minimal action for $\mathrm{S}(y)$ then gives the Euler-Lagrange equation

$$
\frac{\delta L(x, y)}{\delta Z_{v}^{(a)}}-\partial_{\mu} \frac{\delta L(x, y)}{\delta\left(\partial_{\mu} Z_{v}^{(a)}\right)}=0
$$

which in turn leads to the Klein-Gordon equation

$$
\left(\square+m_{Z^{(a)}}^{2}\right) Z_{\mu}^{(a)}(x)=0
$$

for the effective vector field $Z_{\mu}^{(a)}(x)$ defined as

$$
Z_{\mu}^{(a)}(x) \equiv \int \mathrm{d} y Z_{\mu}^{(a)}(x, y)
$$

In this way we obtain

- For $\alpha(a) \neq 0$ :

$$
\left(\square-\eta_{a a} \frac{g_{V_{A}}^{(a)^{2}}}{\alpha(a)}\right) Z_{\mu}^{(a)}=0
$$

And hence

$$
m_{Z^{(a)}}^{2}=-\eta_{a a} \frac{g_{V_{A}}^{(a)^{2}}}{\alpha(a)}
$$

- For $\alpha(a)=0$ :

The Lagrangian (13) has no kinetic term for the field $Z_{\mu}^{(a)}$ which now can be considered as auxiliary field.

So, the conclusion is that a single neutral vector field in space-time with extradimensions leads to a set of (not more than d) effective neutral vector fields in ordinary 4-dimensional space-time with the masses obeying the sum rule followed from (14) and (20):

$$
-\sum_{a}\left\{\eta_{a a} \frac{g_{V_{A}}^{(a)^{2}}}{m_{Z^{(a)}}^{2}}\right\}=1
$$


For illustration let us consider the case $d=2$. In this case a single vector field $V_{M}(x, y)$ can give two effective vector fields $Z_{\mu}^{(1)}(x)$ and $Z_{\mu}^{(1)}(x)$. By putting, for example, $\alpha(1)=\alpha(2)=\frac{1}{2}$ and $\alpha(1)=2, \alpha(2)=-1$,

Equation (20) gives:

$$
m_{Z^{(a)}}^{2}=-2 \eta_{a a} g_{V_{a}}^{2}
$$

and

$$
\begin{aligned}
& m_{Z^{(1)}}^{2}=-\frac{1}{2} \eta_{11} g_{V_{1}}^{2} \\
& m_{Z^{(2)}}^{2}=\eta_{22} g_{V_{2}}^{2}
\end{aligned}
$$

respectively

It is worth noting that by choosing appropriate values of $\alpha(a)$ and Minkowski metric of extradimensions the described formalism could be useful in giving the relation between the metric of extradimensions and tachyons having negative squared mass.

\section{Conclusion}

In this work we extend the results obtained in our previous work [9] to consider the mass spectrum for vector bosons. It has been shown that a single neutral vector field in space-time with $d$ extradimensions corresponds to a set of s $(\leq d)$ effective neutral vector fields in ordinary 4-di- mensional space-time with masses obeying the sum rule expressed in terms of metric of extradimensions and parameter function dictated from the periodicity conditions. This would give a deeper understanding of the relation between extradimensions and gauge bosons in the construction of the Unified Gauge Theory of interactions.

\section{REFERENCES}

[1] C. Cs'aKi, "TASI Lectures on Extradimensions and Branes," hep-ph 0404096, 2004.

[2] L. Randall and M. D. Schwarz, JHEP, 2001.

[3] R. Sundrum, TASI, 2005.

[4] M. B. Green, J. H. Schwarz and E. Witten, "Superstring Theory," Cambridge University Press, Cambridge, 1987.

[5] L. Brink and M. Henneaux, "Principles of String Theory," Plenum Press, New York, 1988. doi:10.1007/978-1-4613-0909-3

[6] G. Furlan, et al., "Superstrings, Supergravity and Unified Thoeries," World Scientific, Singapore, 1997.

[7] L. O'Raifeartaigh and N. Straumann, "Early History of Gauge Theories and Kaluza-Klein Theories," arXiv-hepth /98/05, 1999.

[8] T. G. Rizzo, "Pedagogical Introduction to Extradimensions," arXiv-hepth /0409309, 2005.

[9] D. V. Duc and N. M. Giao, "A Mechanism for Mass Creation from Extradimensions," arXiv-hepth/1301.1405, 2013. 